\title{
A horizontal Chern-Gauss-Bonnet formula on totally geodesic foliations
}

\author{
Fabrice Baudoin ${ }^{1} \cdot$ Erlend Grong $^{2}$ (D) - Gianmarco Vega-Molino ${ }^{1}$
}

Received: 8 July 2021 / Accepted: 8 January 2022 / Published online: 1 February 2022

(c) The Author(s) 2022

\section{Abstract}

Under suitable conditions, we show that the Euler characteristic of a foliated Riemannian manifold can be computed only from curvature invariants which are transverse to the leaves. Our proof uses the hypoelliptic sub-Laplacian on forms recently introduced by two of the authors in Baudoin and Grong (Ann Glob Anal Geom 56(2):403-428, 2019).

\section{Introduction}

The goal of the paper is to prove the following result:

Theorem 1.1 Let $\mathbb{M}$ be a smooth, connected, oriented and $n+m$ dimensional compact manifold. We assume that $\mathbb{M}$ is equipped with a Riemannian foliation $\mathcal{F}$ with bundle-like metric $g$ and totally geodesic m-dimensional leaves. We also assume that the horizontal distribution $\mathcal{H}=\mathcal{F}^{\perp}$ is bracket-generating and that there exists $\varepsilon>0$ such that

$$
\left(\nabla_{v} J\right)_{w}=-\frac{1}{2 \varepsilon}\left[J_{v}, J_{w}\right]
$$

for any $v, w \in T_{x} \mathbb{M}, x \in \mathbb{M}$, where $\nabla$ is the Bott connection of the foliation and $J$ is the tensor defined in (2.2). Denoting $\chi(\mathbb{M})$ the Euler characteristic of $\mathbb{M}$ :

- If $n$ or $m$ is odd, then $\chi(\mathbb{M})=0$;

F. Baudoin: Author supported in part by the NSF Grant DMS 1901315. E. Grong: Author supported by grant from the Trond Mohn Foundation-Grant TMS2021STG02 (GeoProCo).

\section{Erlend Grong}

erlend.grong@uib.no

Fabrice Baudoin

fabrice.baudoin@uconn.edu

Gianmarco Vega-Molino

gianmarco.molino@uconn.edu

1 Department of Mathematics, University of Connecticut, Storrs, CT, USA

2 Department of Mathematics, University of Bergen, P.O. Box 7803, 5020 Bergen, Norway 
- If $n$ and $m$ are both even, then

$$
\chi(\mathbb{M})=\int_{\mathbb{M}} \hat{\omega}_{\mathcal{H}}^{\varepsilon} \wedge\left[\operatorname{det}\left(\frac{\mathscr{T}}{\sinh (\mathscr{T})}\right)^{1 / 2}\right]_{m} .
$$

Notations are further explained in Sect. 4, but we point out that a remarkable feature of that result is that the density $\hat{\omega}_{\mathcal{H}}^{\varepsilon} \wedge\left[\operatorname{det}\left(\frac{\mathscr{T}}{\sinh (\mathscr{T})}\right)^{1 / 2}\right]_{m}$ essentially only depends on horizontal curvature quantities. Therefore, the theorem illustrates further the fact already observed in [4] that topological properties of $\mathbb{M}$ might be obtained from horizontal curvature invariants only provided that the bracket-generating condition of the horizontal distribution is satisfied; thus, in essence, the theorem is a sub-Riemannian result. We also note that the condition (1.1) is satisfied in a large class of examples including the H-type foliations introduced in [5], see Example 2.4.

The proof of Theorem 1.1 is based on the study of the heat semigroup generated by the hypoelliptic sub-Laplacian on forms recently introduced in [4]. The heat equation approach to Chern-Gauss-Bonnet type formulas (or index formulas) that we are using is of course not new: It was suggested by Atiyah-Bott [1] and McKean-Singer [16] and first carried out by Patodi [18] and Gilkey [12] and is by now classical, see the book [9]. However, a difficulty in our setting is that the sub-Laplacian on forms we consider is only hypoelliptic but not elliptic. To carry out the required small-time asymptotics analysis to obtain the horizontal Chern-Gauss-Bonnet formula, we will make use of the probabilistic Brownian Chen series parametrix method first introduced in [3] and which is easy to adapt to hypoelliptic situations, see [2].

The paper is organized as follows. In Sect. 2, we introduce the horizontal Laplacian on forms $\Delta_{\mathcal{H}, \varepsilon}$ and prove that it is a self-adjoint operator if and only if the condition (1.1) is satisfied. In Sect. 3, we prove a McKean-Singer type formula for $\Delta_{\mathcal{H}, \varepsilon}$, namely that for every $t>0$,

$$
\operatorname{Str}\left(e^{t \Delta \mathcal{H}, \varepsilon}\right)=\chi(\mathbb{M})
$$

Finally, in Sect. 4 we study the small-time asymptotics of $\operatorname{Str}\left(e^{t \Delta} \mathcal{H}, \varepsilon\right)$ and conclude the proof of Theorem 1.1.

\section{Preliminaries}

In this section, we first recall the framework and notations of Baudoin and Grong [4] and the references therein to which we refer for further details. We then prove a necessary and sufficient condition for the form horizontal Laplacian of a totally geodesic foliation to be a symmetric operator.

\subsection{Totally geodesic foliations}

Let $(\mathbb{M}, g)$ be a smooth, oriented, connected, compact Riemannian manifold with dimension $n+m$. We assume that $\mathbb{M}$ is equipped with a foliation $\mathcal{F}$ with $m$-dimensional leaves. The distribution $\mathcal{V}$ formed by vectors tangent to the leaves is referred to as the set of vertical directions (or vertical subbundle). Define the horizontal subbundle $\mathcal{H}=\mathcal{V}^{\perp}$ as its orthogonal complement. We will always assume in this paper that the horizontal distribution $\mathcal{H}$ is 
everywhere bracket-generating. The foliation is called Riemannian and totally geodesic if for any $X \in \Gamma(\mathcal{H}), Z \in \Gamma(\mathcal{V})$, the respective conditions are satisfied,

$$
\left(\mathcal{L}_{Z} g\right)(X, X)=0, \quad\left(\mathcal{L}_{X} g\right)(Z, Z)=0 .
$$

Equivalently, we can describe these conditions using the Bott connection. Write $\pi_{\mathcal{H}}$ and $\pi \mathcal{V}$ for the respective orthogonal projections to $\mathcal{H}$ and $\mathcal{V}$. Let $\nabla^{g}$ be the Levi-Civita connection of $g$. Introduce a new connection $\nabla$ on $T \mathbb{M}$ according to the rules,

$$
\nabla_{X} Y= \begin{cases}\pi_{\mathcal{H}}\left(\nabla_{X}^{g} Y\right) & \text { for any } X, Y \in \Gamma(\mathcal{H}), \\ \pi_{\mathcal{H}}([X, Y]) \text { for any } X \in \Gamma(\mathcal{V}), Y \in \Gamma(\mathcal{H}), \\ \pi_{\mathcal{V}}([X, Y]) \text { for any } X \in \Gamma(\mathcal{H}), Y \in \Gamma(\mathcal{V}), \\ \pi_{\mathcal{V}}\left(\nabla_{X}^{g} Y\right) \text { for any } X, Y \in \Gamma(\mathcal{V}) .\end{cases}
$$

We observe that $\nabla$ preserves $\mathcal{H}$ and $\mathcal{V}$ under parallel transport. The foliation $\mathcal{F}$ is then both Riemannian and totally geodesic if and only if $\nabla g=0$. For the rest of the paper, we will assume that $\nabla$ is indeed compatible with the metric $g$. The torsion $T$ of $\nabla$ is given by

$$
T(X, Y)=-\pi_{\mathcal{V}}\left[\pi_{\mathcal{H}} X, \pi_{\mathcal{H}} Y\right] .
$$

Define a corresponding endomorphism valued one-form $Z \mapsto J_{Z}$ by

$$
\left\langle J_{Z} X, Y\right\rangle_{g}=\langle Z, T(X, Y)\rangle_{g}, \quad X, Y, Z \in \Gamma(T \mathbb{M}) .
$$

Let $g_{\mathcal{H}}$ and $g_{\mathcal{V}}$ be the respective restrictions of $g$ to $\mathcal{H}$ and $\mathcal{V}$. We then define the canonical variation $g$ by $g_{\varepsilon}=g_{\mathcal{H}} \oplus \frac{1}{\varepsilon} g_{\mathcal{V}}, \varepsilon>0$, and make the following observations:

(i) If $(\mathbb{M}, \mathcal{F}, g)$ is a Riemannian, totally geodesic foliation, then so is $\left(\mathbb{M}, \mathcal{F}, g_{\varepsilon}\right)$.

(ii) Although the Levi-Civita connection $\nabla^{g_{\varepsilon}}$ of $g_{\varepsilon}$ is different from the connection $\nabla^{g}$ of $g$, replacing $\nabla^{g}$ with $\nabla^{g_{\varepsilon}}$ in formula (2.1) will lead to exactly the same connection. In other words, when defining the Bott connection $\nabla$, we obtain the same connection for any metric $g_{\varepsilon}$ in the family of canonical variations.

(iii) For any fixed $\varepsilon>0$, define a connection

$$
\hat{\nabla}_{X}^{\varepsilon} Y=\nabla_{X} Y+\frac{1}{\varepsilon} J_{X} Y .
$$

This connection preserves $\mathcal{H}$ and $\mathcal{V}$ under parallel transport and is compatible with $g_{\varepsilon^{\prime}}$ for any $\varepsilon^{\prime}>0$. Furthermore, its torsion

$$
\hat{T}^{\varepsilon}(X, Y)=T(X, Y)+\frac{1}{\varepsilon} J_{X} Y-\frac{1}{\varepsilon} J_{Y} X,
$$

is skew-symmetric with respect to $g_{\varepsilon}$. Hence, if we consider its adjoint connection

$$
\nabla_{X}^{\varepsilon} Y=\hat{\nabla}_{X}^{\varepsilon} Y-\hat{T}^{\varepsilon}(X, Y)=\nabla_{X} Y-T(X, Y)+\frac{1}{\varepsilon} J_{Y} X
$$

it will also be compatible with $g_{\varepsilon}$. However, $\mathcal{H}$ and $\mathcal{V}$ are not parallel with respect to $\nabla^{\varepsilon}$.

\subsection{Horizontal Laplacian on forms}

For the totally geodesic Riemannian foliation $(\mathbb{M}, \mathcal{F}, g)$, define its horizontal Laplacian on functions $f \in C^{\infty}(\mathbb{M})$ by

$$
\Delta_{\mathcal{H}} f=\operatorname{tr}_{\mathcal{H}} \nabla_{\times} d f(\times) .
$$


We note that since $\mathcal{H}$ is assumed to be bracket-generating, from Hörmander's theorem, $\Delta_{\mathcal{H}}$ is a subelliptic operator. We also note that since $g_{\mathcal{H}}$ and the Bott connection are independent of $\varepsilon>0$, the horizontal Laplacian is as well; that is, the choice of any metric $g_{\varepsilon}$ in the canonical variation family will not change $g_{\mathcal{H}}$, the Bott connection, or the horizontal Laplacian.

Consider now the totally geodesic Riemannian foliation $\left(\mathbb{M}, \mathcal{F}, g_{\varepsilon}\right)$ for some fixed $\varepsilon>0$. We want to extend the horizontal Laplacian on functions (2.5) to a differential operator on forms $\Delta_{\mathcal{H}, \varepsilon}$ satisfying the following requirements:

(I) $\Delta_{\mathcal{H}, \varepsilon} f=\Delta_{\mathcal{H}} f$ for any smooth function $f$;

(II) The operator $\Delta_{\mathcal{H}, \varepsilon}$ is of Weitzenböck type, i.e., $\Delta_{\mathcal{H}, \varepsilon}=L_{\mathcal{H}, \varepsilon}+\mathscr{R}_{\varepsilon}$ where $\mathscr{R}_{\varepsilon}$ is a zero-order differential operator and

$$
L_{\mathcal{H}, \varepsilon}=\operatorname{tr}_{\mathcal{H}} \tilde{\nabla}_{\times, \times}^{2},
$$

is the connection horizontal Laplacian of some connection $\tilde{\nabla}$ compatible with $g_{\varepsilon}$;

(III) If $d$ is the exterior differential, then

$$
\left[\Delta_{\mathcal{H}, \varepsilon}, d\right]=0 .
$$

Given these requirements, there is an essentially unique extension of $\Delta_{\mathcal{H}}$ to forms, see $[4,15]$ for details. We call $\Delta_{\mathcal{H}, \varepsilon}$ the $\varepsilon$-horizontal Laplacian on forms. This operator can described as follows.

Proposition 2.1 (Horizontal Laplacian on forms, see [4]) Consider the $\varepsilon$-horizontal divergence operator defined by

$$
\delta_{\mathcal{H}, \varepsilon} \eta=-\operatorname{tr}_{\mathcal{H}}\left(\nabla_{\times}^{\varepsilon} \eta\right)(\times, \cdot)
$$

The operator

$$
\Delta_{\mathcal{H}, \varepsilon}=-\delta_{\mathcal{H}, \varepsilon} d-d \delta_{\mathcal{H}, \varepsilon}
$$

is called the $\varepsilon$-horizontal Laplacian on forms, and it satisfies the requirements (I), (II), (III). In particular, this operator has Weitzenböck decomposition $\Delta_{\mathcal{H}, \varepsilon}=L_{\mathcal{H}, \varepsilon}+\mathscr{R}_{\varepsilon}$ where $L_{\mathcal{H}, \varepsilon}$ is defined as in (2.6) relative to $\nabla^{\varepsilon}$.

We can describe the zero order operator $\mathscr{R}_{\varepsilon}$ can be made explicit, see [4]. For later use, we will prefer to write the operators using Fermion calculus, see Appendix A.1. Let $X_{1}, \ldots, X_{n}$ and $Z_{1}, \ldots, Z_{m}$ be local orthonormal bases of, respectively, $\mathcal{H}$ and $\mathcal{V}$. Define $a_{i}=\iota_{X_{i}}$ and $b_{r}=\iota_{Z_{r}}$ for the corresponding annihilation operators, with the dual operators $a_{i}^{*}=X_{i}^{*} \wedge$ and $b_{r}^{*}=Z_{r}^{*} \wedge$ acting by wedge products. The dual are here relative to the $L^{2}$ inner product with respect to the fixed metric $g$. Relative to the curvature tensor $\hat{R}^{\varepsilon}$ of $\hat{\nabla}^{\varepsilon}$, write

$$
\hat{R}_{i j k}^{\varepsilon, l}=\left\langle\hat{R}^{\varepsilon}\left(X_{i}, X_{j}\right) X_{k}, X_{l}\right\rangle_{g},
$$

and use similar notation for other tensors with indices $i, j, k, l$ denoting evaluations with respect to the basis of $\mathcal{H}$, indices $r$, $s$ with respect to the basis of $\mathcal{V}$. We emphasize that these indices are always defined relative to the fixed metric $g$. Then, $\mathscr{R}_{\varepsilon}$ is given by

$$
\begin{aligned}
\mathscr{R}_{\varepsilon}= & \sum_{i, j, k=1}^{n} \hat{R}_{i j k}^{\varepsilon, i} a_{k}^{*} a_{j}+\sum_{i, k=1}^{n} \sum_{r=1}^{m} \hat{R}_{i r k}^{\varepsilon, i} a_{k}^{*} b_{r}+\frac{1}{2} \sum_{i, j, k, l=1}^{n} \hat{R}_{i j k}^{\varepsilon, l} a_{k}^{*} a_{l}^{*} a_{j} a_{i} \\
& +\sum_{i, j, k=1}^{n} \sum_{r=1}^{m} \hat{R}_{i r k}^{\varepsilon, l} a_{k}^{*} a_{l}^{*} b_{r} a_{i}+\frac{1}{2} \sum_{i, j=1}^{n} \sum_{r, s=1}^{m} \hat{R}_{r s i}^{\varepsilon, j} a_{i}^{*} a_{j}^{*} b_{r} b_{s} .
\end{aligned}
$$


We want to give a formula for this operator that shows the dependence of $\varepsilon$ explicitly. Let $T$ and $R$ be the curvature of the Bott connection $\nabla$ and use indices after semi-colons to denote covariant derivatives with respect to this connection. Using Lemma A.2, Appendix, we can write

$$
\begin{aligned}
\mathscr{R}_{\varepsilon}= & \sum_{i, j, k=1}^{n}\left(R_{k j i}^{k}+\frac{1}{\varepsilon} \sum_{r=1}^{m} T_{i k}^{r} T_{j k}^{r}\right) a_{i}^{*} a_{j}-\sum_{i, j=1}^{n} \sum_{r=1}^{m} T_{i j ; i}^{r} a_{j}^{*} b_{r} \\
& +\frac{1}{2} \sum_{i, j, k, l=1}^{n}\left(R_{k l i}^{j}+\frac{1}{\varepsilon} \sum_{r=1}^{m} T_{k l}^{r} T_{i j}^{r}\right) a_{i}^{*} a_{j}^{*} a_{l} a_{k}+\sum_{i, j, k=1}^{n} \sum_{r=1}^{m} \frac{1}{\varepsilon} T_{i j ; k}^{r} a_{i}^{*} a_{j}^{*} b_{r} a_{k} \\
& +\frac{1}{2} \sum_{i, j=1}^{n} \sum_{r, s=1}^{m}\left(\frac{2}{\varepsilon} T_{i j ; r}^{s}+\frac{1}{\varepsilon^{2}} \sum_{k=1}^{n}\left(T_{k j}^{r} T_{i k}^{s}-T_{k j}^{s} T_{i k}^{r}\right)\right) a_{i}^{*} a_{j}^{*} b_{s} b_{r} .
\end{aligned}
$$

\subsection{Symmetry of the horizontal Laplacian}

Consider the exterior algebra

$$
\Omega=\Omega(\mathbb{M})=\bigoplus_{k=0}^{\operatorname{dim} \mathbb{M}} \Omega^{k}
$$

with the $L^{2}$-inner product from $g_{\varepsilon}$. When restricted to elements in $\Omega^{0} \oplus \Omega^{1}$, the operator $\Delta_{\mathcal{H}, \varepsilon}$ is symmetric if and only if $\mathcal{H}$ satisfies the Yang-Mills condition, i.e., if

$$
\sum_{i=1}^{n} T_{i j ; i}^{r}=0, \quad \text { for any } \quad j=1, \ldots, n, r=1, \ldots, m .
$$

see [6]. Considering all forms, we have the following result.

Proposition 2.2 The operator $\Delta_{\mathcal{H}, \varepsilon}$ is symmetric with respect to the $L^{2}$-inner product of $g_{\varepsilon}$ if and only if

$$
\left(\nabla_{v} J\right)_{w}=-\frac{1}{2 \varepsilon}\left[J_{v}, J_{w}\right]
$$

for any $v, w \in T_{x} M, x \in M$. In particular, $\nabla_{v} J=0$ for any $v \in \mathcal{H}$.

We note that under the above condition, the expression of $\mathscr{R}_{\varepsilon}$ reduces to

$$
\mathscr{R}_{\varepsilon}=\sum_{i, j, k=1}^{n}\left(R_{k j i}^{k}+\frac{1}{\varepsilon} \sum_{r=1}^{m} T_{i k}^{r} T_{j k}^{r}\right) a_{i}^{*} a_{j}+\frac{1}{2} \sum_{i, j, k, l=1}^{n}\left(R_{k l i}^{j}+\frac{1}{\varepsilon} \sum_{r=1}^{m} T_{k l}^{r} T_{i j}^{r}\right) a_{i}^{*} a_{j}^{*} a_{l} a_{k} .
$$

Proof $L_{\mathcal{H}, \varepsilon}$ is symmetric by Grong and Thalmaier [15, Lemma A.1], so we only need to determine when $\mathscr{R}_{\varepsilon}$ is symmetric. We choose a local bases $X_{1}, \ldots, X_{n}$ and $Z_{1}, \ldots, Z_{m}$ of, respectively, $\mathcal{H}$ and $\mathcal{V}$. We consider the representation of $\mathscr{R}_{\varepsilon}$ as in (2.9). Then, for $\mathscr{R}_{\varepsilon}$ to be 
symmetric, we must have

$$
\begin{aligned}
0 & =\left\langle\mathscr{R}_{\varepsilon} X_{k}^{*} \wedge Z_{r}^{*}, X_{i}^{*} \wedge X^{j}\right\rangle_{\varepsilon}-\left\langle\mathscr{R}_{\varepsilon} X_{i}^{*} \wedge X_{j}, X_{k}^{*} \wedge Z_{r}^{*}\right\rangle_{\varepsilon}=\frac{1}{\varepsilon} T_{i j ; k}^{r}, \\
0 & =\left\langle\mathscr{R}_{\varepsilon} Z_{r}^{*} \wedge Z_{s}^{*}, X_{i}^{*} \wedge X^{j}\right\rangle_{\varepsilon}-\left\langle\mathscr{R}_{\varepsilon} X_{i}^{*} \wedge X_{j}, Z_{r}^{*} \wedge Z_{s}^{*}\right\rangle_{\varepsilon} \\
& =\frac{2}{\varepsilon} T_{i j ; r}^{s}+\frac{1}{\varepsilon^{2}} \sum_{k=1}^{n}\left(T_{k j}^{r} T_{i k}^{s}-T_{k j}^{s} T_{i k}^{r}\right) .
\end{aligned}
$$

These equations are clearly equivalent to (2.10). If these hold, then $\mathscr{R}_{\varepsilon}$ reduces to the expression (2.11), which is symmetric by Lemma A.3 (i).

Remark 2.3 If we assume that $m=1$ (i.e., the leaves are one-dimensional), then it is immediate from the previous result that the following are equivalent:

(i) $\Delta_{\mathcal{H}, \varepsilon}$ is symmetric for some $\varepsilon>0$.

(ii) $\Delta_{\mathcal{H}, \varepsilon}$ is symmetric for all $\varepsilon>0$.

(iii) $\nabla J=0$.

Recall that the statement $\nabla J=0$ is equivalent to $\nabla T=0$. For $m>1$, the above statement remains true if we replace (i) by the following assumption

(i') $\Delta_{\mathcal{H}, \varepsilon}$ is symmetric at least two values $\varepsilon>0$ and $\varepsilon^{\prime}>0$.

Example 2.4 (H-type foliations) Following definitions given in [5], we say that a foliated Riemannian manifold $(\mathbb{M}, \mathcal{F}, g)$ is of $H$-type if for every $Z \in \Gamma(\mathcal{V})$, we have $J_{Z}^{2}=-\|Z\|_{\mathcal{V}}^{2} \pi_{\mathcal{H}}$. Expand the definition of $J$ from taking values from $\mathcal{V}$ to its $\operatorname{Clifford}$ algebra $\mathbf{C l}(\mathcal{V})$ by the rule $J_{1}=\pi_{\mathcal{H}}$ and iteratively $J_{u \cdot v}=J_{u} J_{v}, u, v \in \mathbf{C l}(\mathcal{V})$. We then further say that the foliation is of horizontally parallel Clifford type if $\nabla_{X} J=0$ for any horizontal vector fields $X \in \Gamma(\mathcal{H})$ and while for $u, v \in \mathcal{V}$.

$$
\left(\nabla_{u} J\right)_{v} \in J_{\mathbf{C l}(\mathcal{V})} .
$$

It then turns out that for some $\kappa \in \mathbb{R}$,

$$
\left(\nabla_{u} J\right)_{v}=-\kappa J_{u \cdot v+\langle u, v\rangle}=-\frac{\kappa}{2}\left[J_{u}, J_{v}\right] .
$$

The number $\kappa$ determines the Ricci curvature of $\nabla$, see [5, Theorem 3.16]. We see that if we have an H-type Riemannian foliation $(\mathbb{M}, \mathcal{F}, g)$ of horizontally parallel Clifford type, then $\Delta_{\mathcal{H}, \varepsilon}$ is symmetric with respect to $g_{\varepsilon}$ for $\varepsilon=\frac{1}{\kappa}$.

Finally, to conclude the section we point out the following result. For the definition of the Carnot-Carathéodory metric $d_{c c}$ of the sub-Riemannian manifold $\left(\mathbb{M}, \mathcal{H}, g_{\mathcal{H}}\right)$ and the tangent cone of a metric space, see, e.g., [13].

Corollary 2.5 Assume that $\Delta_{\mathcal{H}, \varepsilon}$ is symmetric on forms for some fixed $\varepsilon>0$. Then, the following holds:

(a) The horizontal bundle $\mathcal{H}$ has step 2 , that is $\mathcal{H}+[\mathcal{H}, \mathcal{H}]=T \mathbb{M}$. In particular, the torsion $T$ of the Bott connection $\nabla$ will be surjective on $\mathcal{V}$.

(b) The tangent cones of the metric space $\left(\mathbb{M}, d_{c c}\right)$ at any pair of points $x, y \in \mathbb{M}$ are isometric. 
Proof (a) Recall that if $\Delta_{\mathcal{H}, \varepsilon}$ is symmetric on forms for some $\varepsilon>0$, then in particular $\nabla_{v} J=0$ for any $v \in \mathcal{H}$. We can rewrite it as $\nabla_{v} T=0$ for any $v \in \mathcal{H}$ since $\nabla$ is compatible with $g$. Define $\mathcal{H}^{2}=\mathcal{H}+[\mathcal{H}, \mathcal{H}]$ and let $X_{1}, X_{2}, X_{3} \in \Gamma(\mathcal{H})$ be arbitrary. We first see that

$$
T\left(X_{2}, X_{3}\right)=\nabla_{X_{2}} X_{3}-\nabla_{X_{3}} X_{2}-\left[X_{2}, X_{3}\right]=0 \bmod \mathcal{H}^{2},
$$

since $\nabla$ preserves $\mathcal{H}$. Furthermore, by the definition of the Bott connection

$$
\begin{aligned}
{\left[X_{1},\left[X_{2}, X_{3}\right]\right]=} & -\left[X_{1}, T\left(X_{2}, X_{3}\right)\right] \bmod \mathcal{H}^{2}=-\nabla_{X_{1}} T\left(X_{2}, X_{3}\right) \quad \bmod \mathcal{H}^{2} \\
& =-T\left(\nabla_{X_{1}} X_{2}, X_{3}\right)-T\left(X_{2}, \nabla_{X_{1}} X_{3}\right) \quad \bmod \mathcal{H}^{2}=0 \quad \bmod \mathcal{H}^{2} .
\end{aligned}
$$

It follows that $\mathcal{H}$ only generates $\mathcal{H}^{2}$. As we assumed that $\mathcal{H}$ is bracket generating, we have $\mathcal{H}^{2}=T M$.

(b) Since both $\mathcal{H}$ and $\mathcal{H}^{2}=\mathcal{H}+[\mathcal{H}, \mathcal{H}]=T M$ have constant rank, it follows by Mitchell [17] and Bellaïche [8] that the tangent cone at a point $x$ is a Carnot group $G_{x}$. Its Lie algebra $\mathfrak{g}_{x}$ is given by

$$
\mathfrak{g}_{x}=\mathfrak{g}_{x, 1} \oplus \mathfrak{g}_{x, 2}=\mathcal{H}_{x} \oplus T_{x} M / \mathcal{H}_{x}
$$

where $T M / \mathcal{H}_{x}$ is the center, and for $X_{x}, Y_{x} \in \mathcal{H}_{x}=\mathfrak{g}_{x, 1}$ the Lie bracket is defined as

$$
\llbracket X_{x}, Y_{x} \rrbracket=\left.[X, Y]\right|_{x} \bmod \mathcal{H}_{x} .
$$

where $X, Y$ are any pair of vector fields extending this vectors. The Carnot group $G_{x}$ is then the corresponding simply connected Lie group of $\mathfrak{g}_{x}$ with the sub-Riemannian structure given by left translation of $\mathfrak{g}_{x}=\mathcal{H}_{x}$ and its inner product.

If identify $\mathfrak{g}_{x}=\mathcal{H}_{x} \oplus T_{x} M / \mathcal{H}_{x}$ with $T_{x} M=\mathcal{H}_{x} \oplus \mathcal{V}_{x}$ through the map $v \bmod \mathcal{H}_{x} \mapsto$ $\pi \mathcal{V}_{x}(v), v \in T_{x} M$, then the Lie bracket becomes,

$$
\llbracket v, w \rrbracket=-T(v, w), \quad v, w \in T_{x} M .
$$

Let now $y$ be any other point and let $\gamma:[0,1] \rightarrow \mathbb{M}$ be any horizontal curve from $x$ to $y$, which exists form our assumption that $\mathcal{H}$ satisfies the bracket-generating condition. Then, $\nabla_{\dot{\gamma}(t)} T=0$ for any $t \in[0,1]$, so if we write

$$
I_{\gamma, t}=I_{t}: T_{x} \mathbb{M} \rightarrow T_{\gamma(t)} \mathbb{M},
$$

for the parallel transport map along $\gamma$, then this satisfies

$$
I_{t} T(u, v)=T\left(/_{t} u, I_{t} v\right), \quad v, w \in T_{x} \mathbb{M} .
$$

As a consequence, $/_{1}: \mathfrak{g}_{x}=T_{x} \mathbb{M} \rightarrow \mathfrak{g}_{y}=T_{y} \mathbb{M}$ is a Lie algebra isomorphism, which can be integrated to a Lie group isomorphism from $G_{x}$ to $G_{y}$. Since the parallel transport $I_{1}$ also maps $\mathcal{H}_{x}$ onto $\mathcal{H}_{y}$ isometrically, the induced map on Carnot groups is in fact a sub-Riemannian isometry.

\section{Horizontal McKean-Singer theorem}

We work on a totally geodesic foliation $(\mathbb{M}, \mathcal{F}, g)$ and assume that there is some $0<\varepsilon<+\infty$ such that horizontal Laplacian $\Delta_{\mathcal{H}, \varepsilon}$, is symmetric. From Proposition 2.2, this assumption is 
equivalent to the fact that

$$
\left(\nabla_{v} J\right)_{w}=-\frac{1}{2 \varepsilon}\left[J_{v}, J_{w}\right] .
$$

Since $\Delta_{\mathcal{H}, \varepsilon}$ commutes with $d$ on smooth forms and is symmetric, it also commutes on smooth forms with the coderivative $\delta_{\varepsilon}$, and thus, it also commutes with the Hodge-de Rham operator $\Delta_{\varepsilon}:=-d \delta_{\varepsilon}-\delta_{\varepsilon} d$ on smooth forms. From Hodge theorem, the operator $\Delta_{\varepsilon}$ is elliptic with a compact resolvent and the space of $L^{2}$-forms can be decomposed as $\oplus_{k=0}^{+\infty} E_{\lambda_{k}}$ where the $E_{\lambda_{k}}$ 's are the eigenspaces of $\Delta_{\varepsilon}$. Those eigenspaces only contain smooth forms, therefore $\Delta_{\mathcal{H}, \varepsilon}\left(E_{\lambda_{k}}\right) \subset E_{\lambda_{k}}$. This implies that $\Delta_{\mathcal{H}, \varepsilon}$ is essentially self-adjoint and generates the semigroup:

$$
e^{t \Delta} \mathcal{H}, \varepsilon=\oplus_{k=0}^{+\infty} e^{t \Delta} \mathcal{H}, \varepsilon \mid E_{\lambda_{k}}
$$

By hypoellipticity (see [4, Lemma 4.9]), this semigroup has a smooth kernel $p_{\mathcal{H}, \varepsilon}(t, x, y)$ and is a bounded trace class operator in $L_{\mu}^{2}\left(\wedge^{\bullet} \mathbb{M}, g_{\varepsilon}\right)$. Let us denote by $E_{0}^{+}\left(\Delta_{\mathcal{H}, \varepsilon}\right)$ (resp. $\left.E_{0}^{-}\left(\Delta_{\mathcal{H}, \varepsilon}\right)\right)$ the space of harmonic even forms for $\Delta_{\mathcal{H}, \varepsilon}$ (resp. the space of harmonic odd forms for $\left.\Delta_{\mathcal{H}, \varepsilon}\right)$.

The goal of the section is to prove the following theorem, which is an analogue for our horizontal Laplacian of the classical McKean-Singer formula found in [16] :

Theorem 3.1 (Horizontal McKean-Singer formula) For every $t>0$,

$$
\begin{aligned}
\operatorname{Str}\left(e^{t \Delta_{\mathcal{H}, \varepsilon}}\right): & =\int_{\mathbb{M}} \operatorname{Tr}\left(p_{\mathcal{H}, \varepsilon}^{+}(t, x, x)\right) d \mu(x)-\int_{\mathbb{M}} \operatorname{Tr}\left(p_{\mathcal{H}, \varepsilon}^{-}(t, x, x)\right) d \mu(x) \\
& =\operatorname{dim} E_{0}^{+}\left(\Delta_{\mathcal{H}, \varepsilon}\right)-\operatorname{dim} E_{0}^{-}\left(\Delta_{\mathcal{H}, \varepsilon}\right) \\
& =\chi(\mathbb{M})
\end{aligned}
$$

where $\chi(\mathbb{M})$ is the Euler characteristic of $\mathbb{M}$.

We turn to the proof of Theorem 3.1. We denote by

$$
\mathbf{D}_{\varepsilon}=d+\delta_{\varepsilon}
$$

the Dirac operator of the metric $g_{\varepsilon}$. Observe that $\mathbf{D}_{\varepsilon}$ commutes with $\Delta_{\mathcal{H}, \varepsilon}$ since both $d$ and $\delta_{\varepsilon}$ commute with it. The main idea to prove Theorem 3.1 is to introduce a deformation of $\Delta_{\mathcal{H}, \varepsilon}$ as follows:

$$
\square_{\varepsilon, \theta}=(1-\theta) \Delta_{\mathcal{H}, \varepsilon}-\theta \mathbf{D}_{\varepsilon}^{2}, \quad \theta \in[0,1] .
$$

A first lemma is the following:

Lemma 3.2 Let $\lambda$ be a nonzero eigenvalue of $\square_{\varepsilon, \theta}$. Then, $\mathbf{D}_{\varepsilon}: E_{\lambda}^{+}\left(\square_{\varepsilon, \theta}\right) \rightarrow E_{\lambda}^{-}\left(\square_{\varepsilon, \theta}\right)$ is an isomorphism. Therefore, $\operatorname{dim} E_{\lambda}^{+}\left(\square_{\varepsilon, \theta}\right)=\operatorname{dim} E_{\lambda}^{-}\left(\square_{\varepsilon, \theta}\right)$.

Proof Let $\lambda$ be a nonzero eigenvalue of $\square_{\varepsilon, \theta}$. The corresponding eigenspace $E_{\lambda}\left(\square_{\varepsilon, \theta}\right)$ is finite-dimensional since $e^{t \square_{\varepsilon, \theta}}$ is a compact operator for $t>0$. Moreover, since $\mathbf{D}_{\varepsilon}$ commutes with $\square_{\varepsilon, \theta}, \mathbf{D}_{\varepsilon}: E_{\lambda}^{+}\left(\square_{\varepsilon, \theta}\right) \rightarrow E_{\lambda}^{-}\left(\square_{\varepsilon, \theta}\right)$ is well defined. Let now $\alpha \in E_{\lambda}^{+}\left(\square_{\varepsilon, \theta}\right)$ such that $\mathbf{D}_{\varepsilon} \alpha=0$. One has then

$$
d \alpha=-\delta_{\varepsilon} \alpha
$$

This implies that

$$
\|d \alpha\|_{L^{2}\left(\wedge \cdot \mathbb{M}, g_{\varepsilon}\right)}^{2}=-\left\langle d \alpha, \delta_{\varepsilon} \alpha\right\rangle_{L^{2}\left(\wedge \cdot \mathbb{M}, g_{\varepsilon}\right)}=0,
$$


so $d \alpha=0$. Similarly, one has $\left\|\delta_{\varepsilon} \alpha\right\|_{L^{2}\left(\wedge \cdot \mathbb{M}, g_{\varepsilon}\right)}^{2}=0$, so $\delta_{\varepsilon} \alpha=0$. Therefore,

$$
\alpha=\frac{1-\theta}{\lambda} \Delta_{\mathcal{H}, \varepsilon} \alpha=-\frac{1-\theta}{\lambda}\left(d \delta_{\mathcal{H}, \varepsilon}+\delta_{\mathcal{H}, \varepsilon} d\right) \alpha=-\frac{1-\theta}{\lambda} d \delta_{\mathcal{H}, \varepsilon} \alpha
$$

One deduces

$$
\|\alpha\|_{L^{2}\left(\wedge \cdot \mathbb{M}, g_{\varepsilon}\right)}^{2}=-\frac{1-\theta}{\lambda}\left\langle\alpha, d \delta_{\mathcal{H}, \varepsilon} \alpha\right\rangle_{L^{2}\left(\wedge \cdot \mathbb{M}, g_{\varepsilon}\right)}=-\frac{1-\theta}{\lambda}\left\langle\delta_{\varepsilon} \alpha, \delta_{\mathcal{H}, \varepsilon} \alpha\right\rangle_{L^{2}\left(\wedge \cdot \mathbb{M}, g_{\varepsilon}\right)}=0 .
$$

As a consequence, $\mathbf{D}_{\varepsilon}: E_{\lambda}^{+}\left(\square_{\varepsilon, \theta}\right) \rightarrow E_{\lambda}^{-}\left(\square_{\varepsilon, \theta}\right)$ is injective. Let us now prove that it is surjective. Let $\alpha \in E_{\lambda}^{-}\left(\square_{\varepsilon, \theta}\right)$ which is orthogonal to the space $\mathbf{D}_{\varepsilon} E_{\lambda}^{+}\left(\square_{\varepsilon, \theta}\right)$. For every $\omega \in E_{\lambda}^{+}\left(\square_{\varepsilon, \theta}\right)$, one has

$$
0=\left\langle\alpha, \mathbf{D}_{\varepsilon} \omega\right\rangle_{L^{2}\left(\wedge \cdot \mathbb{M}, g_{\varepsilon}\right)}=\left\langle\mathbf{D}_{\varepsilon} \alpha, \omega\right\rangle_{L^{2}\left(\wedge \cdot \mathbb{M}, g_{\varepsilon}\right)} .
$$

Thus, $\mathbf{D}_{\varepsilon} \alpha=0$ and from the first part of the proof, we deduce that $\alpha=0$. We conclude that $\mathbf{D}_{\varepsilon}: E_{\lambda}^{+}\left(\square_{\varepsilon, \theta}\right) \rightarrow E_{\lambda}^{-}\left(\square_{\varepsilon, \theta}\right)$ is indeed an isomorphism.

A second lemma is the following:

Lemma 3.3 For every $t>0$, the map $\theta \rightarrow \operatorname{Str}\left(e^{t \square_{\varepsilon, \theta}}\right)$ is continuous on $[0,1]$.

Proof Let $q_{\varepsilon, \theta}(t, x, y)$ be the heat kernel of $\square_{\varepsilon, \theta}=(1-\theta) \Delta_{\mathcal{H}, \varepsilon}-\theta \mathbf{D}_{\varepsilon}^{2}, p_{\mathcal{H}, \varepsilon}(t, x, y)$ be the heat kernel of $\Delta_{\mathcal{H}, \varepsilon}$ and $p_{\varepsilon}(t, x, y)$ be the heat kernel of $-\mathbf{D}_{\varepsilon}^{2}$. Since $-\mathbf{D}_{\varepsilon}^{2}$ and $\Delta_{\mathcal{H}, \varepsilon}$ commute, we have

$$
e^{t \square_{\varepsilon, \theta}}=e^{t(1-\theta) \Delta \mathcal{H}, \varepsilon} e^{-t \theta \mathbf{D}_{\varepsilon}^{2}}
$$

Therefore:

$$
q_{\varepsilon, \theta}(t, x, y)=\int_{\mathbb{M}} p_{\mathcal{H}, \varepsilon}(t(1-\theta), x, z) p_{\varepsilon}(t \theta, z, y) d z
$$

and the result easily follows since

$$
\operatorname{Str}\left(e^{t \square_{\varepsilon, \theta}}\right)=\int_{\mathbb{M}} q_{\varepsilon, \theta}(t, x, x) d x .
$$

We are now ready for the proof of Theorem 3.1.

Proof From the first lemma:

$$
\begin{aligned}
& \operatorname{Str}\left(e^{t \square_{\varepsilon, \theta}}\right) \\
& \quad=\operatorname{dim} E_{0}^{+}\left(\square_{\varepsilon, \theta}\right)-\operatorname{dim} E_{0}^{-}\left(\square_{\varepsilon, \theta}\right)+\sum_{\lambda \neq 0}\left(\operatorname{dim} E_{\lambda}^{+}\left(\square_{\varepsilon, \theta}\right)-\operatorname{dim} E_{\lambda}^{-}\left(\square_{\varepsilon, \theta}\right)\right) e^{\lambda t} \\
& \quad=\operatorname{dim} E_{0}^{+}\left(\square_{\varepsilon, \theta}\right)-\operatorname{dim} E_{0}^{-}\left(\square_{\varepsilon, \theta}\right) .
\end{aligned}
$$

Therefore, $\operatorname{Str}\left(e^{t \square_{\varepsilon, \theta}}\right) \in \mathbb{Z}$. From the second lemma, $\theta \rightarrow \operatorname{Str}\left(e^{t \square_{\varepsilon, \theta}}\right)$ is continuous, thus constant. We deduce

$$
\operatorname{Str}\left(e^{t \square_{\varepsilon, 0}}\right)=\operatorname{Str}\left(e^{t \square_{\varepsilon, 1}}\right) .
$$

Since $\square_{\varepsilon^{*}, 1}=-\mathbf{D}_{\varepsilon}^{2}$ is the Hodge-de Rham Laplacian of the Riemannian manifold (M, $g_{\varepsilon}$ ), from the usual Riemannian Hodge theory (see [16]), we have

$$
\operatorname{Str}\left(e^{t \square_{\varepsilon, 1}}\right)=\chi(\mathbb{M}),
$$

4 which concludes the proof. 
Remark 3.4 (Dependence on the symmetry condition) It would obviously be beneficial to prove the above statement without the assumption of symmetry on $\Delta_{\mathcal{H}, \varepsilon}$. A semigroup approach to non-symmetric horizontal Laplacians has been used, see [15, Appendix A]. In the above proof, however, we really rely on the fact that $\Delta_{\mathcal{H}, \varepsilon}$ commutes with the codifferential $\delta_{\varepsilon}$, and with the Laplace-Beltrami operator $-\mathbf{D}_{\varepsilon}^{2}$. We can no longer use these properties if we remove the symmetry assumption.

\section{Horizontal Chern-Gauss-Bonnet formula}

As before, we consider the horizontal Laplacian

$$
\Delta_{\mathcal{H}, \varepsilon}=-d \delta_{\mathcal{H}, \varepsilon}-\delta_{\mathcal{H}, \varepsilon} d,
$$

and assume that it is symmetric for a fixed $\varepsilon$. As seen earlier, $\Delta_{\mathcal{H}, \varepsilon}$ satisfies the Weitzenböck identity

$$
\Delta_{\mathcal{H}, \varepsilon}=L_{\mathcal{H}, \varepsilon}-\mathscr{R}_{\varepsilon}=-\left(\nabla_{\mathcal{H}}^{\varepsilon}\right)^{*} \nabla_{\mathcal{H}}^{\varepsilon}-\mathscr{R}_{\varepsilon} .
$$

where the later equality follows from [15, Lemma 2.1]. The goal of the section is to compute the pointwise limit

$$
\lim _{t \rightarrow 0} \operatorname{Str}\left(p_{\mathcal{H}, \varepsilon}(t, x, x)\right)
$$

and deduce from it our horizontal Chern-Gauss-Bonnet formula. The computation of that limit will be based on the probabilist method of Brownian Chen series (see [3,7]) which has the advantage of being easily adapted to subelliptic operators like $\Delta_{\mathcal{H}, \varepsilon}$, see [2]. For convenience and to introduce notation, we include in Appendix A.2 the main elements of that theory.

A first step to implement the method in [2] is to study the small-time heat kernel asymptotics of a diffusion tangent to the scalar horizontal Laplacian $\Delta_{\mathcal{H}}$. Since we assume that $\Delta_{\mathcal{H}, \varepsilon}$ is symmetric, from Corollary 2.5 one has $T \mathbb{M}=\mathcal{H}+[\mathcal{H}, \mathcal{H}]$, and thus the tangent diffusion will take its values in a two-step Carnot group [the so-called tangent cone, see Corollary 2.5(b)] for which an explicit formula for the heat kernel is known (see [10,11]). In a local horizontal frame $\left\{X_{1}, \ldots, X_{n}\right\}$ around $x_{0}$ write

$$
V_{t}\left(x_{0}\right)=\sum_{i=1}^{n} \sqrt{2} X_{i}\left(x_{0}\right) B_{t}^{i}+\sum_{1 \leq i<j \leq n} \pi \mathcal{V}\left(\left[X_{i}, X_{j}\right]\left(x_{0}\right)\right) \int_{0}^{t} B_{s}^{i} d B_{s}^{j}-B_{s}^{j} d B_{s}^{i},
$$

where $\left(B_{t}\right)_{t \geq 0}$ is a Brownian motion in $\mathbb{R}^{n}$. We note that $V_{t}\left(x_{0}\right)$ can be written in a basis free way as

$$
\sqrt{2} B_{t}\left(x_{0}\right)-\int_{0}^{t} T\left(B_{s}\left(x_{0}\right), d B_{s}\left(x_{0}\right)\right)
$$

where $B_{t}\left(x_{0}\right)=\sum_{i=1}^{n} X_{i}\left(x_{0}\right) B_{t}^{i}$ is a standard Brownian motion in $\mathcal{H}_{x_{0}}$.

Lemma 4.1 Let $x_{0} \in \mathbb{M}$. For $t>0$, let $d_{t}\left(x_{0}\right)$ be the density at 0 of the $T_{x_{0}} \mathbb{M}$ valued random variable $V_{t}\left(x_{0}\right)$. Then, when $t \rightarrow 0$,

$$
d_{t}\left(x_{0}\right) \sim \frac{2^{m}}{(4 \pi t)^{\frac{n}{2}+m}} \int_{\mathcal{V}_{x_{0}}} \operatorname{det}\left(\frac{\sqrt{J_{z}^{*} J_{z}}}{\sinh \sqrt{J_{z}^{*} J_{z}}}\right)^{1 / 2} d z
$$


Proof The process $\left(V_{t}\left(x_{0}\right)\right)_{t \geq 0}$ is the horizontal Brownian motion in the tangent cone $G_{x_{0}}$ which is a 2-step Carnot group when it is identified with $T_{x_{0}} \mathbb{M}$ using the group exponential map. The heat kernel of the horizontal Laplacian is known explicitly in 2-step Carnot groups (see $[10,11])$ which yields the small-time asymptotics.

Remark 4.2 We note that $d_{t}\left(x_{0}\right)$ is independent of $x_{0}$ because of Corollary 2.5(b).

In the sequel, we will use the notation $\mathcal{F}_{I}$ (defined with respect to the connection $D=\nabla^{\varepsilon}$ ) and $\Lambda_{I}(B)_{t}$, as introduced and discussed in Appendix A.2.

Corollary 4.3 It will hold that as $t \rightarrow 0$

$$
\operatorname{Str}\left(p_{\mathcal{H}, \varepsilon}\left(t, x_{0}, x_{0}\right)\right) \sim d_{t}\left(x_{0}\right) \mathbb{E}\left(\operatorname{Str}\left(\exp \left(\sum_{I, d(I) \leq n+2 m} \Lambda_{I}(B)_{t} \mathcal{F}_{I}\right)\left(x_{0}\right)\right) \mid B_{1}=0\right)
$$

where $d_{t}\left(x_{0}\right)$ is the density at 0 of $V_{t}(x)$, as in Lemma 4.1.

Proof Since $\mathcal{H}$ is two-step bracket generating, the homogeneous dimension is $Q=\operatorname{dim} \mathcal{H}+$ $2 \operatorname{dim} \mathcal{V}=n+2 m$. Taking $N=n+2 m$ in Theorem A.1, and applying similar arguments as in the proof of Proposition 4.2 in [3], the corollary follows by recognizing that for $|I|>2, X_{I}$ is a linear combination of $X_{i},\left[X_{j}, X_{k}\right]$ so that when $t \rightarrow 0$ the density at 0 of

$$
\sum_{I, d(I) \leq n+2 m} \Lambda_{I}(B)_{t} X_{I}
$$

is equivalent to $d_{t}\left(x_{0}\right)$ from the previous lemma.

Applying the previous results, we are now able to compute $\lim _{t \rightarrow 0} \operatorname{Str}\left(p_{\mathcal{H}, \varepsilon}\left(t, x_{0}, x_{0}\right)\right)$. Choose local orthonormal bases $X_{1}, \ldots, X_{n}$ and $Z_{1}, \ldots, Z_{m}$ of, respectively, $\mathcal{H}$ and $\mathcal{V}$.

Lemma 4.4 The integral

$$
\mathcal{J}=\mathcal{J}\left(x_{0}\right)=\frac{2^{m}}{(2 \pi)^{\frac{n}{2}+m}} \int_{\mathcal{V}_{x_{0}}} \operatorname{det}\left(\frac{\sqrt{J_{z}^{*} J_{z}}}{\sinh \sqrt{J_{z}^{*} J_{z}}}\right)^{1 / 2} d z
$$

is a constant, so independent of the point $x_{0} \in \mathbb{M}$ chosen. Furthermore, it holds that

$$
\lim _{t \rightarrow 0} \operatorname{Str}\left(p_{\mathcal{H}, \varepsilon}\left(t, x_{0}, x_{0}\right)\right)=\left\{\begin{array}{l}
\frac{\mathcal{J}}{\left(\frac{n}{2}+m\right) !} \mathbb{E}\left(\operatorname{Str}\left[A_{x_{0}}^{\frac{n}{2}+m}\right] \mid B_{1}=0\right), \text { if } n \text { is even } \\
0, \quad \text { if } n \text { is odd } .
\end{array}\right.
$$

where the random variable $A_{x_{0}}$ is given by

$$
\begin{aligned}
A_{x_{0}}=- & \frac{1}{2} \sum_{i, j, k, l=1}^{n}\left(R_{k l i}^{j}+\frac{1}{\varepsilon} \sum_{r=1}^{m} T_{k l}^{r} T_{i j}^{r}\right) a_{i}^{*} a_{j}^{*} a_{l} a_{k} \\
& , \sum_{1 \leq i<j \leq n} \sum_{r, s=1}^{m} T_{i j ; r}^{s} b_{r}^{*} b_{s} \int_{0}^{1} B_{t}^{i} d B_{t}^{j}-B_{t}^{j} d B_{t}^{i} .
\end{aligned}
$$


Proof First, observe that

$$
\mathcal{J}\left(x_{0}\right)=(2 t)^{\frac{n}{2}+m} d_{t}\left(x_{0}\right),
$$

and so the independence of $\mathcal{J}\left(x_{0}\right)$ from $x_{0}$ follows from Corollary 2.5(b) as in Remark 4.2.

Consider the expansion

$$
\operatorname{Str}\left[\exp \left(\sum_{I, d(I) \leq n+2 m} \Lambda_{I}(B)_{t} \mathcal{F}_{I}\right)\left(x_{0}\right)\right]=\sum_{k \geq 0} \frac{1}{k !} \operatorname{Str}\left[\left(\sum_{I, d(I) \leq n+2 m} \Lambda_{I}(B)_{t} \mathcal{F}_{I}\right)^{k}\left(x_{0}\right)\right] .
$$

From the Weitzenböck identity (4.1), we have for $i, j \in\{1, \ldots, n+m\}$ that

$$
\mathcal{F}_{0}=-\mathscr{R}_{\varepsilon}, \quad \mathcal{F}_{i}=0, \quad \mathcal{F}_{(i, j)}=\hat{R}^{\varepsilon}\left(Y_{i}, Y_{j}\right)
$$

where $\left\{Y_{1}, \ldots, Y_{n+m}\right\}$ form a local orthonormal frame and the $\left\{c_{i}, c_{i}^{*}\right\}_{i=1}^{n+m}$ form the associated Fermion calculus of $T \mathbb{M}$. Equation (2.11) allows us to write

$$
\mathscr{R}_{\varepsilon}=\sum_{i, j, k=1}^{n}\left\langle\hat{R}^{\varepsilon}\left(X_{i}, X_{k}\right) X_{j}, X_{i}\right\rangle_{g} a_{k}^{*} a_{i}+\sum_{i, j, k, l}\left\langle\hat{R}^{\varepsilon}\left(X_{i}, X_{j}\right) X_{k}, X_{l}\right\rangle_{g} a_{i}^{*} a_{j}^{*} a_{l} a_{k}
$$

where $\left\{a_{i}, a_{i}^{*}\right\}$ form the Fermion calculus for $\mathcal{H}$.

Recalling equation (A.1) in the appendix, we see that the supertrace will vanish for any term that is not of full degree; from our expressions for $\mathcal{F}_{I}$, it is thus clear that for $k<\frac{n}{2}+m$

$$
\operatorname{Str}\left[\left(\sum_{I, d(I) \leq n+2 m} \Lambda_{I}(B)_{t} \mathcal{F}_{I}\right)^{k}\left(x_{0}\right)\right]=0 .
$$

Let us assume that $n$ is even. Applying the scaling property of Brownian motion, when $t \rightarrow 0$ the term $k=\frac{n}{2}+m$ will be dominant. More precisely,

$$
\begin{aligned}
& \mathbb{E}\left(\operatorname{Str}\left[\exp \left(\sum_{I, d(I) \leq n+2 m} \Lambda_{I}(B)_{t} \mathcal{F}_{I}\right)\left(x_{0}\right)\right] \mid B_{1}=0\right) \\
& =\frac{1}{\left(\frac{n}{2}+m\right) !} \mathbb{E}\left(\operatorname{Str}\left[\left(\sum_{I, d(I) \leq n+2 m} \Lambda_{I}(B)_{t} \mathcal{F}_{I}\right)^{\frac{n}{2}+m}\left(x_{0}\right)\right] \mid B_{1}=0\right)+O\left(t^{\frac{n}{2}+m+\frac{1}{2}}\right) .
\end{aligned}
$$

Then, we have,

$$
\begin{aligned}
& \mathbb{E}\left(\operatorname{Str}\left[\left(\sum_{I, d(I) \leq n+2 m} \Lambda_{I}(B)_{t} \mathcal{F}_{I}\right)^{\frac{n}{2}+m}\left(x_{0}\right)\right] \mid B_{1}=0\right) \\
& \quad=\mathbb{E}\left(\operatorname{Str}\left[\left(-t \mathscr{R}_{\varepsilon}\left(x_{0}\right)+\sum_{1 \leq i<j \leq n} \sum_{r, s=1}^{s} \hat{R}_{i i r}^{\varepsilon, s} b_{r}^{*} b_{s} \int_{0}^{t} B_{u}^{i} d B_{u}^{j}-B_{u}^{j} d B_{u}^{i}\right)^{\frac{n}{2}+m}\right] B_{1}=0\right)+O\left(t^{\frac{n}{2}+m+\frac{1}{2}}\right)(4 .
\end{aligned}
$$

We can further simplify this expression using that by Lemma A.2, Appendix, we know that $\hat{R}_{i j r}^{\varepsilon, s}=R_{i j r}^{s}=T_{i j ; r}^{s}$. We also use (2.11) and the fact that only the last term in $\mathscr{R}_{\varepsilon}$ contributes to the supertrace. Combining Lemma 4.1, Corollary 4.3, and Eqs. (4.3) and (4.4), we apply the scaling property of Brownian motion again to find

$$
\begin{aligned}
& \operatorname{Str}\left(p_{\mathcal{H}, \varepsilon}\left(t, x_{0}, x_{0}\right)\right)=\frac{\mathcal{J}}{\left(\frac{n}{2}+m\right) !} \mathbb{E}\left(\operatorname{Str}\left[A_{x_{0}}^{\frac{n}{2}+m}\right] \mid\right. \\
& \left.B_{1}=0\right)+O\left(t^{\frac{1}{2}}\right) .
\end{aligned}
$$

If $n$ is odd, we get by similar arguments that

$$
\operatorname{Str}\left(p_{\mathcal{H}, \varepsilon}\left(t, x_{0}, x_{0}\right)\right)=O\left(t^{\frac{1}{2}}\right) .
$$


completing the proof.

In what follows, we will introduce the tensor $\mathscr{T}$ by

$$
\mathscr{T}\left(Y_{1}, Y_{2}\right)=\hat{R}^{\varepsilon}\left(\pi_{\mathcal{H}} Y_{1}, Y_{2}\right) \pi_{\mathcal{V}}=\pi_{\mathcal{V}} \hat{R}^{\varepsilon}\left(\pi_{\mathcal{H}} Y_{1}, Y_{2}\right) .
$$

We observe that for any $X_{1}, X_{2} \in \Gamma(\mathcal{H})$ and $Z \in \mathcal{V}$,

$$
\mathscr{T}\left(X_{1}, X_{2}\right) Z=\left(\nabla_{Z} T\right)\left(X_{1}, X_{2}\right)=\frac{1}{2 \varepsilon}\left(T\left(J_{Z} X_{1}, X_{2}\right)+T\left(X_{1}, J_{Z} X_{2}\right)\right),
$$

where the latter equality follows from the symmetry condition of $\Delta_{\mathcal{H}, \varepsilon}$.

Example 4.5 (H-type foliation) We again consider the case of the of H-type foliations as in Example 2.4. We recall that in this case, we have that $\Delta_{\mathcal{H}, \varepsilon}$ for $\varepsilon=\frac{1}{\kappa}$. Let $x \in \mathbb{M}$ be a fixed point and let $\mathbf{C l}\left(\mathcal{V}_{x}\right)$ be the Clifford algebra of the vertical space. We remark that in this case, for any $u, v \in \mathcal{H}_{x}$ with $v \in\left(\operatorname{span}_{\zeta \in \mathbf{C l}\left(\mathcal{V}_{x}\right)} J_{\zeta} u\right)^{\perp}$, we have $\mathscr{T}(u, v)=0$. On the other hand, if $v=J_{\zeta} u$, then for any $z \in \mathcal{V}_{x}$,

$$
\mathscr{T}\left(u, J_{\zeta} u\right) z=\kappa \pi \mathcal{V}_{x}\left(z \cdot \zeta^{\text {odd }}\right),
$$

where $\zeta^{\text {odd }}$ is the odd part of $\zeta$ and $\pi \mathcal{V}_{x} \mathrm{Cl}\left(\mathcal{V}_{x}\right) \rightarrow \mathcal{V}_{x}$ is the projection to the first-order part.

We can use the above definition and the previous lemma to prove the following.

Proposition 4.6 Assume that $n$ or $m$ is odd, then

$$
\lim _{t \rightarrow 0} \operatorname{Str}\left(p_{\mathcal{H}, \varepsilon}(t, x, x)\right) d x=0
$$

Assume that both $n$ and $m$ are even, then

$$
\lim _{t \rightarrow 0} \operatorname{Str}\left(p_{\mathcal{H}, \varepsilon}(t, x, x)\right) d x=\hat{\omega}_{\mathcal{H}}^{\varepsilon} \wedge\left[\operatorname{det}\left(\frac{\mathscr{T}}{\sinh (\mathscr{T})}\right)^{1 / 2}\right]_{m}
$$

where $[\cdot]_{m}$ denotes the $m$-form part and $\hat{\omega}_{\mathcal{H}}^{\varepsilon}$ is the horizontal Euler form, locally defined as

$$
\hat{\omega}_{\mathcal{H}}^{\varepsilon}=\frac{(-1)^{n / 2} m !}{2^{n / 2}\left(\frac{n}{2}+m\right) !} \mathcal{J} \sum_{\sigma, \tau \in \mathfrak{S}_{n}} \epsilon(\sigma) \epsilon(\tau) \prod_{i=1}^{n-1} \hat{R}_{\sigma(i) \sigma(i+1) \tau(i)}^{\varepsilon, \tau(i+1)} d x_{\mathcal{H}},
$$

In the above formula, $\mathfrak{S}_{n}$ is the set of the permutations of the indices $\{1, \ldots, n\}, \epsilon$ the signature of a permutation, $\hat{R}_{i j k}^{\varepsilon, l}$ is as in (2.7) and $d x_{\mathcal{H}}$ the $n$-form $X_{1}^{*} \wedge \cdots \wedge X_{n}^{*}$.

Proof We first assume that both $n$ and $m$ are even. It remains to compute $\mathbb{E}\left(\operatorname{Str}\left[A_{x_{0}}^{\frac{n}{2}+m}\right] \mid B_{1}=0\right)$. Looking at (4.2), we have

$$
\begin{aligned}
& \mathbb{E}\left(\operatorname{Str}\left[A_{x_{0}}^{\frac{n}{2}+m}\right] \mid B_{1}=0\right) \\
& \quad=\operatorname{Str}\left[\left(-\sum_{i, j, k, l}\left\langle\hat{R}^{\varepsilon}\left(X_{i}, X_{j}\right) X_{k}, X_{l}\right\rangle_{g} a_{i}^{*} a_{j}^{*} a_{l} a_{k}\right)^{n / 2} \mathbb{E}\left[\left(\sum_{1 \leq i<j \leq n} \mathscr{T}\left(X_{i}, X_{j}\right)\left(x_{0}\right) \int_{0}^{1} B_{s}^{i} d B_{s}^{j}-B_{s}^{j} d B_{s}^{i}\right)^{m} \mid B_{1}=0\right]\right]
\end{aligned}
$$

The term $\left(\sum_{i, j, k, l}\left\langle\hat{R}^{\varepsilon}\left(X_{i}, X_{j}\right) X_{k}, X_{l}\right\rangle_{g} a_{i}^{*} a_{j}^{*} a_{l} a_{k}\right)^{n / 2}$ is then analyzed as in the proof of Proposition 5.6 in [7] (see also Lemma 2.35 in [19]) and up to constant yields the horizontal Euler form $\hat{\omega}_{\mathcal{H}}^{\varepsilon}$. On the other hand, using again the formula for the supertrace, the term

$$
\mathbb{E}\left[\left(\sum_{1 \leq i<j \leq n} \mathscr{T}\left(X_{i}, X_{j}\right)\left(x_{0}\right) \int_{0}^{1} B_{s}^{i} d B_{s}^{j}-B_{s}^{j} d B_{s}^{i}\right)^{m} \mid B_{1}=0\right]
$$


can be replaced with

$$
m ! \mathbb{E}\left[\exp \left(\sum_{1 \leq i<j \leq n} \mathscr{T}\left(X_{i}, X_{j}\right)\left(x_{0}\right) \int_{0}^{1} B_{s}^{i} d B_{s}^{j}-B_{s}^{j} d B_{s}^{i}\right) \mid B_{1}=0\right]
$$

and is analyzed using the Lévy area formula as in the proof of Theorem 4.3 in [3]: it yields the top degree Fermionic piece of $\operatorname{det}\left(\frac{\mathscr{T}}{\sinh (\mathscr{T})}\right)^{1 / 2}\left(x_{0}\right) \in \mathbf{E n d}\left(\wedge \mathcal{V}_{x_{0}}^{*}\right)$ (Fermionic calculus is done here on $\mathcal{V}_{x_{0}}$ ).

If $n$ is even and $m$ is odd, a similar analysis shows that

$$
\mathbb{E}\left(\operatorname{Str}\left[A_{x_{0}}^{\frac{n}{2}+m}\right] \mid B_{1}=0\right)=0 .
$$

Combining Theorem 3.1 and Proposition 4.6 finally yields our main theorem:

Theorem 4.7 Assume that both $n$ and $m$ are even, then

$$
\chi(\mathbb{M})=\int_{\mathbb{M}} \hat{\omega}_{\mathcal{H}}^{\varepsilon} \wedge\left[\operatorname{det}\left(\frac{\mathscr{T}}{\sinh \mathscr{T}}\right)^{1 / 2}\right]_{m} .
$$

Assume that $n$ or $m$ is odd, then $\chi(\mathbb{M})=0$.

As a corollary, since $\nabla J=0$ implies $\mathscr{T}=0$, we obtain the following result:

Corollary 4.8 Assume that $\nabla J=0$, then $\chi(\mathbb{M})=0$.

Funding Open access funding provided by University of Bergen (incl Haukeland University Hospital).

Open Access This article is licensed under a Creative Commons Attribution 4.0 International License, which permits use, sharing, adaptation, distribution and reproduction in any medium or format, as long as you give appropriate credit to the original author(s) and the source, provide a link to the Creative Commons licence, and indicate if changes were made. The images or other third party material in this article are included in the article's Creative Commons licence, unless indicated otherwise in a credit line to the material. If material is not included in the article's Creative Commons licence and your intended use is not permitted by statutory regulation or exceeds the permitted use, you will need to obtain permission directly from the copyright holder. To view a copy of this licence, visit http://creativecommons.org/licenses/by/4.0/.

\section{A Appendices}

\section{A.1 Fermion calculus and supertraces}

In this section, we recall some basic elements of Fermion calculus, see section 2.2.2 in [19] for more details. Let $V$ be a $d$-dimensional Euclidean vector space. We denote $V^{*}$ its dual and $\wedge V^{*}=\bigoplus_{k \geq 0} \wedge^{k} V^{*}$, its exterior algebra. If $u \in V^{*}$, we denote $a_{u}^{*}$ the map $\wedge V^{*} \rightarrow \wedge V^{*}$, such that $a_{u}^{*}(\omega)=u \wedge \omega$. The dual map is denoted $a_{u}$. Let now $\theta_{1}, \ldots, \theta_{d}$ be an orthonormal basis of $V^{*}$. We denote $a_{i}=a_{\theta_{i}}$. If $I$ and $J$ are two words with $1 \leq i_{1}<\cdots<i_{k} \leq d$ and $1 \leq j_{1}<\cdots<j_{l} \leq d$, we denote

$$
A_{I J}=a_{i_{1}}^{*} \cdots a_{i_{k}}^{*} a_{j_{1}} \cdots a_{j_{l}} .
$$


The family of all the possible $A_{I J}$ forms a basis of the $2^{2 d}$-dimensional vector space End $\left(\wedge V^{*}\right)$.

If $A \in \operatorname{End}\left(\wedge V^{*}\right)$, the supertrace $\operatorname{Str}(A)$ is the difference of the trace of $A$ on even forms minus the trace of $A$ on odd forms. If $A=\sum_{I, J} c_{I J} A_{I J}$, then we have

$$
\operatorname{Str}(A)=(-1)^{\frac{d(d-1)}{2}} c_{\{1, \ldots, d\}\{1, \ldots, d\}} .
$$

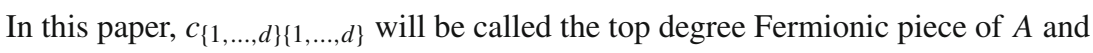

$$
[A]_{d}:=(-1)^{\frac{d(d-1)}{2}} c_{\{1, \ldots, d\}\{1, \ldots, d\}} \theta_{1} \wedge \cdots \wedge \theta_{d}
$$

the $d$-form part of $A$.

\section{A.2 The Brownian Chen series parametrix method}

For the sake of completeness and to introduce some notations used in the paper, we reproduce here the essential ideas from $[2,3,7]$ to which we refer for further details. Let $\mathcal{E}$ be a finitedimensional vector bundle over a compact manifold $\mathbb{M}$ equipped with a connection $D$ and consider a second-order differential operator $\mathcal{L}=D_{0}+\sum_{i=1}^{d} D_{i}^{2}$ with $D_{i}=\mathcal{F}_{i}+D_{X_{i}}$ for some smooth vector fields $X_{i}$ and potentials $\mathcal{F}_{i}$ on $\mathcal{E}$. It is known that the differential equation

$$
\frac{\partial \Phi}{\partial t}=\mathcal{L} \Phi, \quad \Phi(0, x)=f(x)
$$

has solution

$$
\Phi(t, x)=\left(e^{t \mathcal{L}} f\right)(x)=P_{t} f(x) .
$$

At strongly regular points $x_{0} \in \mathbb{M}$, it is furthermore true that $P_{t}$ admits a smooth heat kernel

$$
\begin{aligned}
& p_{t}\left(x_{0}, \cdot\right): \mathbb{R}_{>0} \rightarrow \Gamma(\mathbb{M}, \operatorname{Hom}(\mathcal{E})) \\
& t \mapsto p_{t}\left(x_{0}, \cdot\right)
\end{aligned}
$$

which is to say

$$
\left(P_{t} f\right)\left(x_{0}\right):=\left(e^{t \mathcal{L}} f\right)\left(x_{0}\right)=\int_{\mathbb{M}} p_{t}\left(x_{0}, y\right) f(y) d y .
$$

We have a method of approximation for the heat kernel in this setting.

Theorem A.1 Let $N \geq 1$ and define $\left(P_{t}^{N} f\right)(x)=\mathbb{E}(\Psi(1, x))$ where $\Psi(\tau, x)$ solves the random differential equation

$$
\frac{\partial \Psi}{\psi \tau}=\sum_{I: d(I) \leq N} \Lambda_{I}(B)_{t}\left(D_{I} \Psi\right)(\tau, x), \quad \Psi(0, x)=f(x) .
$$

where $I=\left(i_{1}, \ldots, i_{k}\right) \in\{0, \ldots, d\}^{k}$ is a word, $D_{I}=\left[D_{i_{1}},\left[\ldots,\left[D_{i_{k-1}}, D_{i_{k}}\right] \ldots\right]\right], d(I)=$ $n(I)+k$ with $n(I)$ the number of 0 's in $I$, and the random coefficients are defined by

$$
\Lambda_{I}(B)_{t}=2^{d(I) / 2} \sum_{\sigma \in \mathfrak{S}_{k}} \frac{(-1)^{e(\sigma)}}{k^{2}\left(\begin{array}{c}
k-1 \\
e(\sigma)
\end{array}\right)} \int_{\Delta^{k}[0, t]} \circ d B^{\sigma^{-1}(I)}
$$

where $\left(B_{t}\right)_{t \geq 0}$ is a standard Brownian motion in $\mathbb{R}^{d}$. Then, 
- For $k \geq 0$, define the norm

$$
\|f\|_{k}=\sup _{0 \leq l \leq k} \sup _{0 \leq i_{1}, \ldots, i_{k}} \sup _{x \in \mathbb{M}}\left\|D_{i_{1}} \cdots D_{i_{l}} f(x)\right\| .
$$

It will hold that for any $k \geq 0$

$$
\left\|P_{t} f-P_{t}^{N} f\right\|_{k}=O\left(t^{\frac{N+1}{2}}\right), \quad t \rightarrow 0
$$

- $P_{t}^{N}$ admits a smooth kernel $p_{t}^{N}$ such that for $N \geq 2$

$$
p_{t}\left(x_{0}, x_{0}\right)=p_{t}^{N}\left(x_{0}, x_{0}\right)+O\left(t^{\frac{N+1-Q}{2}}\right), \quad t \rightarrow 0
$$

where $Q$ is the homogeneous dimension at $x_{0}$.

- Write $\mathcal{F}_{I}=D_{I}-D_{X_{I}}$. For $N \geq 2$, it holds as $t \rightarrow 0$ that

$$
\begin{aligned}
& p_{t}^{N}\left(x_{0}, x_{0}\right) \\
& =d_{t}^{N}\left(x_{0}\right) \mathbb{E}\left(\exp \left(\sum_{I, d(I) \leq N} \Lambda_{I}(B)_{t} \mathcal{F}_{I}\right)\left(x_{0}\right) \mid \sum_{I, d(I) \leq N} \Lambda_{I}(B)_{t} X_{I}\left(x_{0}\right)=0\right)+O\left(t^{\frac{N+1-Q}{2}}\right)
\end{aligned}
$$

where $d_{t}^{N}(x)$ is the density at 0 of the random variable $\sum_{I, d(I) \leq N} \Lambda_{I}(B)_{t} X_{I}(x)$.

We refer to Baudoin [2] and Baudoin [7, Section 5.1] for the proofs and further details, but we remark that roughly the theorem says that in small time we can approximate the heat kernel of $\mathcal{L}$ by the kernel associated with solutions of Eq. (A.2), for which we will be able to say much more.

\section{A.3 Curvature of the connection $\hat{\nabla}^{\varepsilon}$}

We want to give details on writing the curvatures of $\hat{\nabla}^{\varepsilon}$ in terms of the Bott connection $\nabla$.

Lemma A.2 Relative to the notation of (2.7) we have the following identities. Recall that $i, j, k, l$ denotes vector fields from a basis of $\mathcal{H}$, while indices $r, s$ denotes such elements from a basis of $\mathcal{V}$

(i) $R_{i j k}^{l}=R_{k l i}^{j}, R_{r_{1} s_{1} r_{1}}^{s_{2}}=R_{r_{2} s_{2} r_{1}}^{s_{1}}$,

(ii) $R_{i j r}^{s}=T_{i j ; r}^{s}, R_{i r k}^{l}=0, R_{i s_{1} r_{2}}^{s_{2}}=0$,

(iii) $T_{i j ; r}^{r}=0$. Equivalently $\left(\nabla_{Z} J\right)_{Z}=0$ for any vector field $Z$ with values in $\mathcal{V}$.

(iv) $\hat{R}_{i j k}^{\varepsilon, l}=R_{i j k}^{l}+\frac{1}{\varepsilon} \sum_{s=1}^{m} T_{i j}^{s} T_{k l}^{s}$.

(v) $\hat{R}_{i r k}^{\varepsilon, l}=\frac{1}{\varepsilon} T_{k l ; i}^{s}$.

(vi) $\hat{R}_{r s k}^{\varepsilon, l}=\frac{2}{\varepsilon} T_{k l ; r}^{s}+\frac{1}{\varepsilon^{2}} \sum_{i=1}^{n}\left(T_{i l}^{r} T_{k i}^{s}-T_{i l}^{s} T_{k i}^{r}\right)$

Proof From (2.3), we observe that

$$
\begin{aligned}
\hat{R}^{\varepsilon}(X, Y) Z=R(X, Y) Z+\frac{1}{\varepsilon}\left(\nabla_{X} J\right)_{Y} Z-\frac{1}{\varepsilon}\left(\nabla_{Y} J\right)_{X} Z \\
+\frac{1}{\varepsilon} J_{T(X, Y)} Z+\frac{1}{\varepsilon^{2}}\left[J_{X}, J_{Y}\right] Z .
\end{aligned}
$$

We will also use the first Bianchi identity for connections with torsion

$$
\circlearrowright R(X, Y) Z=\circlearrowright\left(\nabla_{X} T\right)(X, Y)+\circlearrowright T(T(X, Y), Z),
$$

where $\circlearrowright$ denotes the cyclic sum. We furthermore observe the following identities. 
(i) Since $\left\langle T\left(Y_{1}, Y_{2}\right), Y_{3}\right\rangle$ and $T\left(T\left(Y_{1}, Y_{2}\right), Y_{3}\right)$ vanishes if $Y_{1}, Y_{2}, Y_{3}$ are either all vertical or all horizontal,

$$
\begin{aligned}
\left\langle R\left(X_{1}, X_{2}\right) X_{3}, X_{4}\right\rangle_{g} & =\left\langle R\left(X_{3}, X_{4}\right) X_{1}, X_{2}\right\rangle_{g}, \\
\left\langle R\left(Z_{1}, Z_{2}\right) Z_{3}, Z_{4}\right\rangle_{g} & =\left\langle R\left(Z_{3}, Z_{4}\right) Z_{1}, Z_{2}\right\rangle_{g},
\end{aligned}
$$

for any $X_{i} \in \Gamma(\mathcal{H}), Z_{i} \in \Gamma(\mathcal{V}), i=1,2,3,4$.

(ii) From Grong [14, Appendix A], we know that for $X_{1}, X_{2} \in \Gamma(\mathcal{H}), Z_{1}, Z_{2} \in \Gamma(\mathcal{V})$,

$$
R\left(X_{1}, X_{2}\right) Z_{1}=\left(\nabla_{Z_{1}} T\right)\left(X_{1}, X_{2}\right), \quad R\left(X_{1}, Z_{1}\right) X_{2}=0 \quad R\left(X_{1}, Z_{1}\right) Z_{2}=0 .
$$

(iii) Since $\nabla$ is compatible with the metric then $\left(\nabla_{Z} J\right)_{Z}=0$ for any $Z \in \Gamma(\mathcal{V})$, as for any $X_{1}, X_{2} \in \Gamma(\mathcal{H})$,

$$
\begin{aligned}
0=\left\langle Z, R\left(X_{1}, X_{2}\right) Z\right\rangle_{g} & =\left\langle Z, \circlearrowright R\left(X_{1}, X_{2}\right) Z\right\rangle_{g} \\
& =\left\langle Z,\left(\nabla_{Z} T\right)\left(X_{1}, X_{2}\right)\right\rangle_{g}=\left\langle X_{2},\left(\nabla_{Z} J\right)_{Z} X_{1}\right\rangle_{g} .
\end{aligned}
$$

(iv) We observe first that from (A.3), for any $X_{1}, X_{2}, X_{3}, X_{4} \in \Gamma(\mathcal{H})$

$$
\begin{aligned}
\left\langle\hat{R}^{\varepsilon}\left(X_{1}, X_{2}\right) X_{3}, X_{4}\right\rangle_{g} & =\left\langle R\left(X_{1}, X_{2}\right) X_{3}, X_{4}\right\rangle_{g}+\frac{1}{\varepsilon}\left\langle J_{T\left(X_{1}, X_{2}\right)} X_{3}, X_{4}\right\rangle_{g} \\
& \stackrel{(\mathrm{i})}{=}\left\langle R\left(X_{3}, X_{4}\right) X_{1}, X_{2}\right\rangle_{g}+\frac{1}{\varepsilon}\left\langle T\left(X_{1}, X_{2}\right), T\left(X_{3}, X_{4}\right)\right\rangle_{g} .
\end{aligned}
$$

(v) Next, for any $X_{1}, X_{2} \in \Gamma(\mathcal{H}), Z \in \Gamma(\mathcal{V})$,

$$
\hat{R}^{\varepsilon}\left(X_{1}, Z\right) X_{2} \stackrel{\text { (ii) }}{=} \frac{1}{\varepsilon}\left(\nabla_{X_{1}} J\right)_{Z} X_{2} .
$$

(vi) For the final property observe that

$$
R\left(Z_{1}, Z_{2}\right) X_{1} \stackrel{(\mathrm{ii})}{=} \circlearrowright R\left(Z_{1}, Z_{2}\right) X_{1}=0 .
$$

Hence,

$$
\begin{aligned}
\hat{R}^{\varepsilon}\left(Z_{1}, Z_{2}\right) X_{1} & =\frac{1}{\varepsilon}\left(\nabla_{Z_{1}} J\right)_{Z_{2}} X_{1}-\frac{1}{\varepsilon}\left(\nabla_{Z_{2}} J\right)_{Z_{1}} X_{1}+\frac{1}{\varepsilon^{2}}\left[J_{Z_{1}}, J_{Z_{2}}\right] X_{1} \\
& \stackrel{\text { (iii) }}{=} \frac{2}{\varepsilon}\left(\nabla_{Z_{1}} J\right)_{Z_{2}} X_{1}+\frac{1}{\varepsilon^{2}}\left[J_{Z_{1}}, J_{Z_{2}}\right] X_{1} .
\end{aligned}
$$

\section{References}

1. Atiyah, M.F., Bott, R.: A Lefschetz fixed point formula for elliptic complexes. I. Ann. Math. 2(86), 374-407 (1967)

2. Baudoin, F.: A Bismut type theorem for subelliptic heat semigroups. C. R. Math. Acad. Sci. Paris 344(12), 765-768 (2007)

3. Baudoin, F.: Brownian Chen series and Atiyah-Singer theorem. J. Funct. Anal. 254(2), 301-317 (2008)

4. Baudoin, F., Grong, E.: Transverse Weitzenböck formulas and de Rham cohomology of totally geodesic foliations. Ann. Glob. Anal. Geom. 56(2), 403-428 (2019)

5. Baudoin, F., Grong, E., Molino, G., Rizzi, L.: H-type foliations. arXiv preprint arXiv:1812.02563 (2018)

6. Baudoin, F., Kim, B., Wang, J.: Transverse Weitzenböck formulas and curvature dimension inequalities on Riemannian foliations with totally geodesic leaves. Commun. Anal. Geom. 24(5), 913-937 (2016)

7. Baudoin, F.: Stochastic Taylor expansions and heat kernel asymptotics. ESAIM:PS 16, $453-478$ (2012)

8. Bellaïche, A.: The tangent space in sub-Riemannian geometry. In: Sub-Riemannian geometry, Volume 144 of Progr. Math., pp. 1-78. Birkhäuser, Basel (1996) 
9. Berline, N., Getzler, E., Vergne, M.: Heat kernels and Dirac operators. Grundlehren Text Editions. Springer, Berlin (2004). Corrected reprint of the 1992 original

10. Cygan, J.: Heat kernels for class 2 nilpotent groups. Studia Math. 64(3), 227-238 (1979)

11. Garofalo, N., Tralli, G.: Heat kernels for a class of hybrid evolution equations. arXiv e-print arXiv:2007.10862 (2020)

12. Gilkey, P.B.: Curvature and the eigenvalues of the Laplacian for elliptic complexes. Adv. Math. 10, 344-382 (1973)

13. Gromov. M.: Carnot-Carathéodory spaces seen from within. In: Sub-Riemannian geometry, Volume 144 of Progr. Math., pp. 79-323. Birkhäuser, Basel (1996)

14. Grong, E.: Affine connections and curvature in sub-Riemannian geometry. arXiv e-prints arXiv:2001.03817 (2020)

15. Grong, E., Thalmaier, A.: Stochastic completeness and gradient representations for sub-Riemannian manifolds. Potential Anal. 51(2), 219-254 (2019)

16. McKean, H.P., Jr., Singer, I.M.: Curvature and the eigenvalues of the Laplacian. J. Differ. Geom. 1(1), 43-69 (1967)

17. Mitchell, J.: On Carnot-Carathéodory metrics. J. Differ. Geom. 21(1), 35-45 (1985)

18. Patodi, V.K.: An analytic proof of Riemann-Roch-Hirzebruch theorem for Kaehler manifolds. J. Differ. Geom. 5, 251-283 (1971)

19. Rosenberg, S.: The Laplacian on a Riemannian manifold. Volume 31 of London Mathematical Society Student Texts. Cambridge University Press, Cambridge (1997). An introduction to analysis on manifolds

Publisher's Note Springer Nature remains neutral with regard to jurisdictional claims in published maps and institutional affiliations. 